\title{
Assessing Subjective Emotional Experience in a Non-Autistic Population
}

\author{
Iain Mccaw ${ }^{1,}$,, Sarah Talari ${ }^{2}$ \\ ${ }^{1}$ General Adult Psychiatry, East Riding Addictions Service, Hull, United Kingdom \\ ${ }^{2}$ Learning Disability Service, Parkside Lodge, Leeds, United Kingdom
}

Email address:

imccaw@nhs.net(I. Mccaw), s.talari@nhs.net(S. Talari)

${ }^{*}$ Corresponding author

To cite this article:

Iain McCaw, Sarah Talari. Assessing Subjective Emotional Experience in a Non-Autistic Population. Science Journal of Clinical Medicine. Vol. 8, No. 4, 2019, pp. 39-43. doi: 10.11648/j.sjcm.20190804.12

Received: May 14, 2019; Accepted: June 18, 2019; Published: August 16, 2019

\begin{abstract}
Clinicians within the Leeds Autism Diagnostic Service (LADS) have found that many of our patients have difficulty answering questions pertaining to subjective emotional experience. It was felt by clinicians within the service that service users who were later diagnosed as neuro-typical also had difficulty answering questions related to their subjective emotional state. It was felt that by examining responses to the meaning of four common emotional states (relaxation, anger, anxiety and happiness) in a neuro-typical population, themes or common words or phrases could be elicited in the description of each emotion. These questions were similar in scope to those found within the Autism Diagnostic Observation Schedule, $2^{\text {nd }}$ Edition, which is used by LADS during diagnostic assessments. This would then allow clinicians a means of comparing a service user's responses with what could be expected from a neuro-typical population. We felt that other teams within our organization unconnected with the Autism Diagnostic Service, could serve as a useful neuro-typical population. An online survey link was therefore sent to different administration teams within Leeds and York Partnership Foundation Trust, which allowed participants to complete four questions, each one asking them to describe the subjective meaning of relaxation, anger, anxiety and happiness in turn. There were no specific inclusion or exclusion criteria for these survey participants. Each question provided an area where each survey participant could express this in up to a few sentences. Following this, the results were analysed for common descriptors and actions, associated in the responses for a particular emotion. When analysing the results, it was evident that actions and activities were more frequently used in responses by the survey participants in responses to questions related to relaxation, anger and happiness, however were less apparent in the responses to describe anxiety. It is hoped that these results will be able to guide clinicians in interpreting and scoring diagnostic screening tools for Autism by providing more knowledge about neuro-typical responses.
\end{abstract}

Keywords: Autism, Alexithymia, Neuro-typical, Subjective Emotional Experience

\section{Introduction}

In his book Emotions in Social Psychology, Gerrard Parrott describes how emotions and social psychology are deeply intertwined [1]. He argues that emotions are essentially social, citing examples of jealousy in relationships or anger at others' blameworthy actions. He further describes how the phenomena of social psychology themselves are often emotional, such as aggression linked with anger or hostility and close relationships with attraction and love.

The most recognisable features of autism are those relating to difficulties in social interaction and communication. People with Autism often present to diagnostic services when their inability to associate with others (be it family, colleagues or peers) and to participate in everyday social life (public transport, employment, education, social gatherings) reaches a peak or culminates in crisis. As they wrestle through the changing social milieu around them and encounter unanticipated predicaments, they often are overwhelmed and decompensate rapidly.

The examination of autistic people's emotions along with their perception of others emotions is therefore an integral part of the Autism assessment process, where validated tools 
such as the ADI-R and ADOS are used [2-3]. The ADOS-2 (Autism Diagnostic Interview Schedule) is used in many settings as a gold standard Autism Diagnostic tool, often in combination with the ADI-R (Autism Diagnostic InterviewRevised). ADOS-2, Module 4 is used for verbally fluent adults when making clinical multidisciplinary decisions on diagnosis in the Leeds Autism Diagnostic Service.

During the course of clinical practice using the ADOS-2, clinicians in Leeds have reflected that the part of the ADOS-2 assessment, where the person's subjective description of a range of typical emotions is explored, could be particularly difficult to answer, even for those without Autism. The questions used in this context relate to a person's articulation of different common emotional states.

Clinicians within Leeds Autism Service have noted that those who are diagnosed with Autism struggle to express their subjective emotional state. However, clinicians have also observed that these questions were often difficult for those patients who did not receive an Autism diagnosis, so it was felt that having a greater understanding of typical answers from a non-Autism population (neuro-typical) would help not only in arriving at an unequivocal consensus but also in a better interpretation of interviewees answers during assessments. It was felt that foreknowledge of typical answers would aid in better scoring and thereby improve clinical effectiveness.

\section{Method}

A survey was created to closely reflect questions around basic emotions assessed during the Clinical Decisions Meeting using the ADOS-2. The questions include a person's description of four different emotions-happiness, anger, sadness and relaxation.

These questions included:

a) How would you articulate to others what relaxation means to you?

b) How would you articulate to others what anger means to you?

c) How would you articulate to others what anxiety means to you?

d) How would you articulate to others what happiness means to you?

A cohort of administrative staff within the Trust were asked to complete the survey (which highlighting the fact that it was aimed at people without Autism in its description). There were no formal inclusion or exclusion criteria for this. We received 30 responses which were then evaluated.

Common themes in the description of the four different emotions in the questionnaire were identified.

\section{Results}

The results for the survey produced some interesting findings. There was a wide range in the responses seen however consistent themes, particularly regarding commonly used adjectives were noted for each of the four questions. Actions and activities were commonly used in responses by the survey participants in responses to questions related to relaxation, anger and happiness, however were less apparent in the responses to describe anxiety.

\section{Relaxation}

One of the most common descriptions appeared to be an absence of stress. Adjectives such as peaceful and calm were also used frequently. Reflecting modern terminology, the phrase "chilling out" was used frequency when describing associated actions. Another action that featured frequently was watching TV.

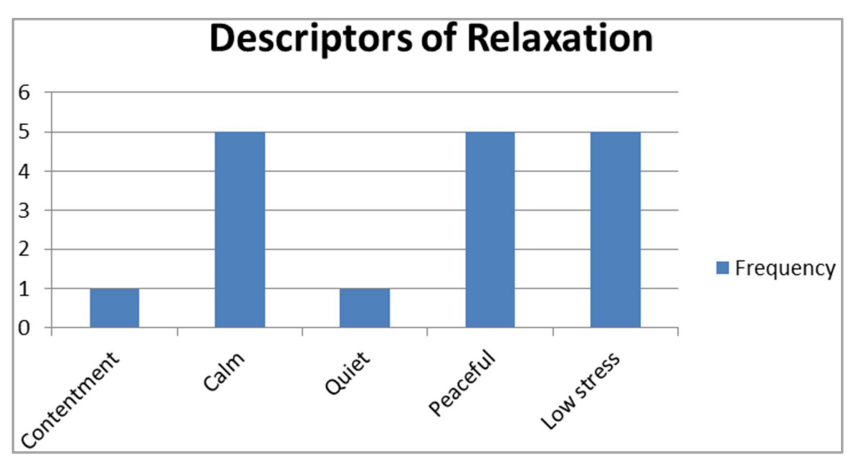

Figure 1. Common adjectives used to describe relaxation.

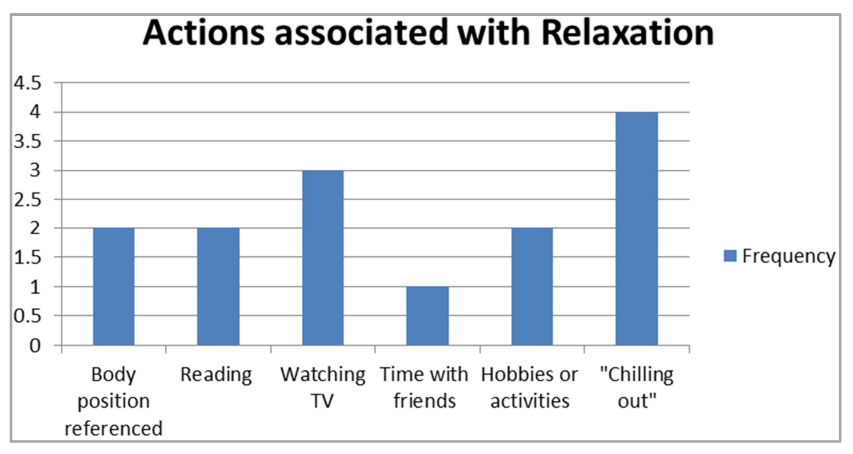

Figure 2. Common respondents associated with relaxation.

\section{Anger}

Anger was notable in that there was one term, used as an adjective and noun that was used above all the others. This was a feeling or a sense of frustration. This was mentioned in 9 of the 30 responses in total, nearly 30 in total. However there was a wide range of other terms that were used. Other terms that featured included negative and stressed. In terms of associated actions, this most frequently included shouting.

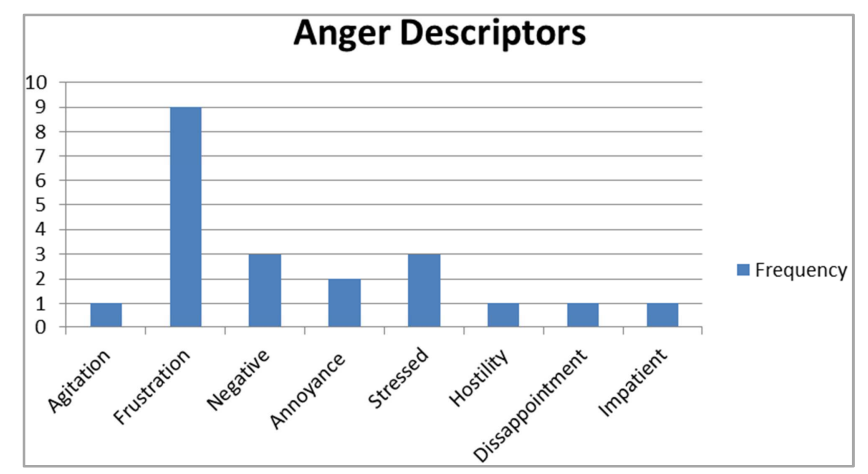

Figure 3. Terms used by respondents when describing anger. 


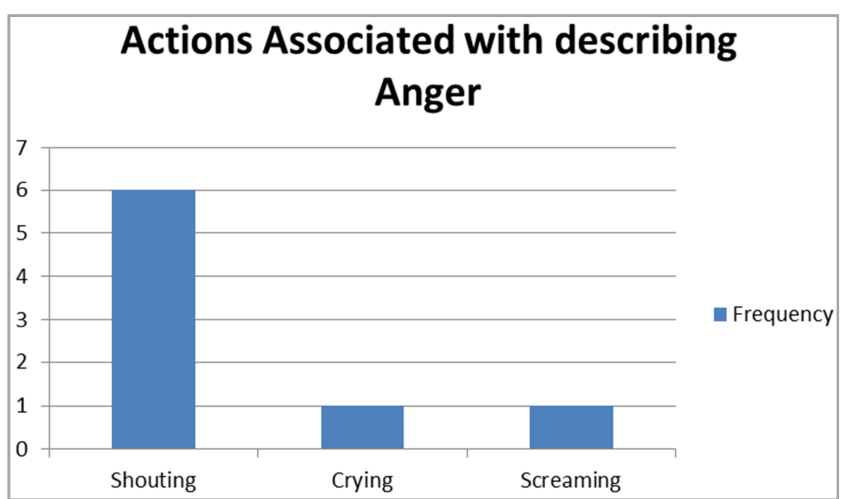

Figure 4. Common actions associated with anger.

Anxiety

Notably for anxiety, responses were focused more on emotions and feelings, rather than specific actions. One of the most frequent nouns and adjective used was worry, featuring in a third of responses. As expected, responses for anxiety frequently referred to associated physical symptoms, which we understand as being due to noradrenaline's effect within the body, the so-called fight or flight response. This understanding forms the basis for Cognitive Behavioural Therapy.

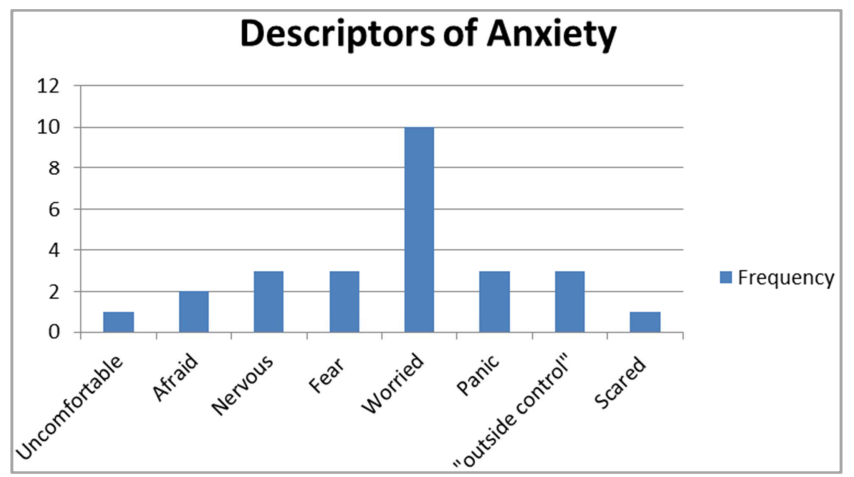

Figure 5. Common terms and emotions associated with anxiety.

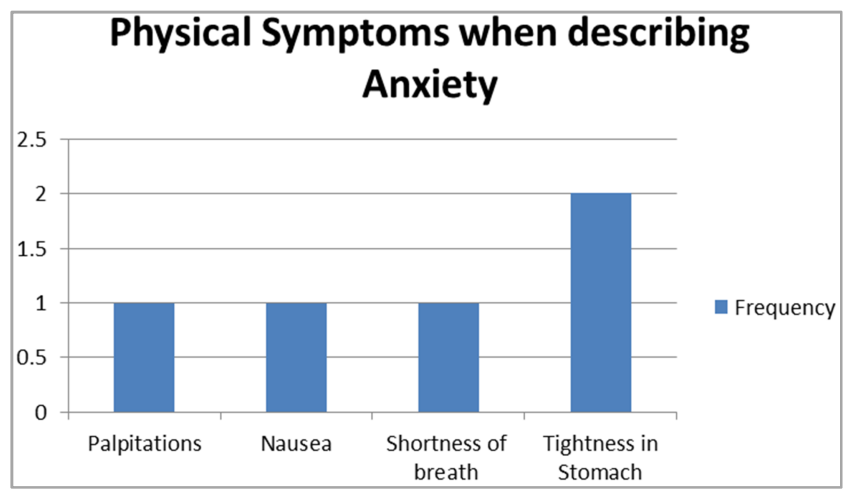

Figure 6. Physical symptoms associated with anxiety in respondents.

\section{Happiness}

The most common descriptors for happiness included contentment as well as feeling good, feelings of warmth and positivity. Happiness was strongly associated with respondents associating activities and actions with how they felt, most commonly being "around friends and family" for 13 out of 30 responses and "smiling" being used in 9 responses.

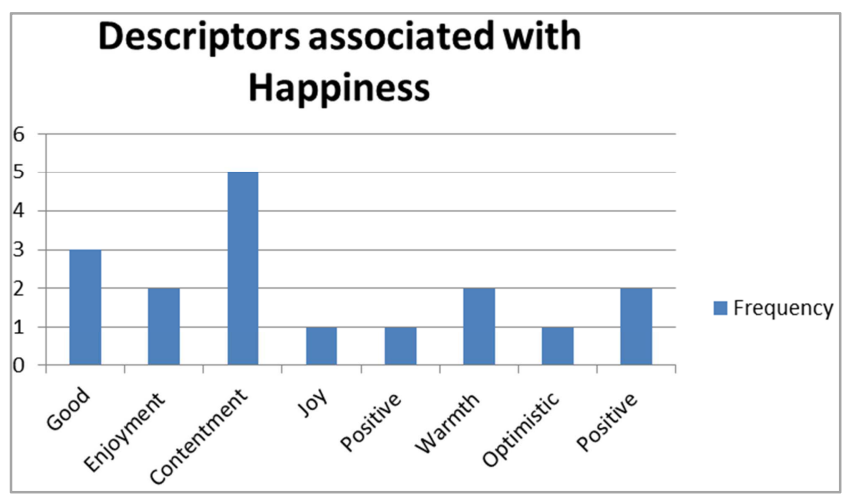

Figure 7. Common terms associated with happiness by respondents.

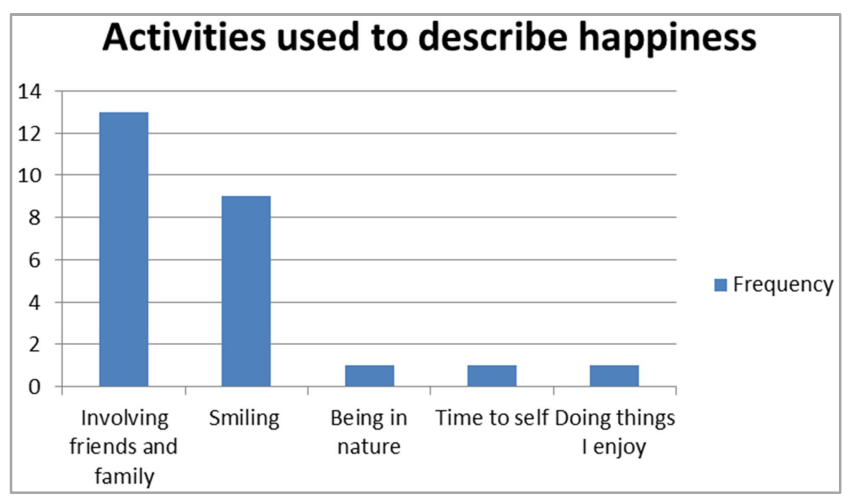

Figure 8. Activities respondents associated with happiness.

\section{Discussions}

Individuals with autism are known to have difficulties describing their subjective emotional state, which is known as Alexithymia [4]. In Alexithymia it is hypothesized that there is poor awareness of the feelings that accompany particular emotions and confusion of emotional and bodily feelings [5]. This also included communication of affective responses [4]. In both ASD and Alexithymia there are thought to be widely overlapping difficulties related to social skills and emotional competence [5]. It has also been shown that Alexithymia, rather than ASD, predicted a lack of expressive response to emotionally charged stimuli [6]. Alexithymia is thought to arise as there is impaired interoception (the ability to perceive the internal state of one's body) and it had been shown that these signals have less impact upon decision making in those with Autistic Spectrum [7].

A higher proportion of individuals with ASD have evidence of Alexithymia, when compared to a control population [8]. It also appears unclear as to whether Alexithymia is a cause or consequence of ASD itself [5]. It is important to highlight however that those with Autism do not lack emotions. However, it has been shown that individuals with ASD rely less on emotional biases in their decision making [9]. It has been hypothesized that those with 
Alexithymia maybe aware they are experiencing an emotion however they are not certain what this could be [10].

There appears to be conflicting evidence as to how individuals with ASD can recognize and respond to the emotional states of others [11-12]. One study indicated that those with Autistic Spectrum Disorder show higher levels of emotional interest when it relates to circumscribed interests, rather than social imagery [13]. An experiment comparing an autistic group of children against those with language disorder and intellectual disability, (to control for differences in intellectual and verbal ability) found that those with autism had greater difficulty and made more errors identifying emotions [14]. Another study compared those an autistic group, with an IQ of 70 and above, with a group of typically developing controls and found no difference between the two groups in terms of their ability to respond to distress [12].

There is some evidence of these abnormal cognitive affective responses on functional MRI, with an Autistic population having less activity in their limbic system compared to a control population, a region of the brain associated with emotional processing [15]. In terms of structural changes, it has been found that there is a reduction in size in the insula, amygdala, orbitofrontal cortex and striatum in those with Alexithymia [16]. It has also been shown that there is reduced activation in the insula, when patients with Alexithymia are involved in tasks which require theory of mind, which may explain some of the overlap with Autism [17].

\section{Conclusions}

Given this knowledge of Alexithymia and how commonly it presents in those with ASD, our results therefore act as a useful comparison as to what descriptors of common emotional states, in a population with typical development, might include or comment on. It would be expected that an individual presenting with ASD would lack some of the emotional descriptors used in our results. Our results therefore could help the judgement of clinicians when scoring the ADOS or taking a screening history.

Our study does have a number of limitations. Firstly, it is a relatively small number of individuals surveyed and therefore this may limit the generalizability of these responses as typical for the wider population. This study does not take account of the effect of mental illness or past trauma and how they may affect the ability of a neurotypical individual to subjectively express their emotional state. Due to issues concerning copyright, our questions were not taken directly from the ADOS questionnaire but were felt to be similar in their purpose and scope. As the survey involved responding in a "white space", in some responses there was ultimately information that had to be omitted when trying to identify common descriptors or themes. Given the wide variety of responses, there could now be scope for a wider ranging questionnaire which further looks at feelings, activities and bodily sensations that are associated with each emotion.

Acknowledgements: Alison Stansfield, lead Consultant for
Leeds Autism Diagnostic Service, has been involved in the supervision of the authors in their preparation and submission of this article. This article has also been reviewed by Conor Davidson and Alwyn Kam, Consultant and Speciality Doctor respectively in the Leeds Autism Diagnostic Service.

\section{References}

[1] Parrot G. Emotions in Social Psychology: Essential Readings. Printed by Edwards Brothers, Ann Arbor, Michigan 2001.

[2] Autism Diagnostic Interview Revised. Rutter, Le Coulter, Lord. Hogrefe, 2003.

[3] Lord et al. Autism Diagnostic Interview Schedule, Second Edition: ADOS-2. Pearson Clinical, 2012.

[4] Moseley et al. Lost for emotion words: What motor and limbic brain activity reveals about autism and semantic theory. NeuroImage 104 (2015) 413-422.

[5] Poquerusse $\mathrm{J}$ et al 2018. Alexithymia and Autism Spectrum Disorder: A complex relationship. Frontiers in Psychology. 9 (1196) 1-10.

[6] Trevisan et al. Alexithymia, but not Autism Spectrum Disorder, maybe related to the production of emotional facial expressions. Molecular Autism (2016) 7: 46.

[7] Shah P, Catmur C, Bird G. Emotional decision-making in autism spectrum disorder: the roles of Interoception and Alexithymia. Shah et al. Molecular Autism (2016) 7: 43.

[8] Bethoz S, Hill EL. The validity of using self-reports to assess emotion regulation abilities in adults with autism spectrum disorder. European Psychiatry Volume 20, Issue 3, May 2005, Pages 291-298.

[9] Brewer et al. The impact of Autism Spectrum Disorder and Alexithymia on Judgements of Moral Acceptability. Journal of Abnormal Psychology, 2015, Vol 124, No. 3, 589-595.

[10] Gaigg S, 2014. People with Autism don't lack emotions but often have difficulty identifying them. $\mathrm{http}: / /$ theconversation.com/people-with-autism-dont-lackemotions-but-often-have-difficulty-identifying-them-25225.

[11] Song Y, Hakonda Y. Selective Impairment of Basic Emotional Recognition in People with Autism: Discrimination Thresholds for Recognition of Facial Expressions of Varying Intensities. Journal of Autism and Developmental Disorders (2018) 48: 1886-1894.

[12] Newbigin et al. Brief Report: Empathic Responsiveness of High Functioning Children with Autism to Expressed and Anticipated Distress. J Autism Dev Disord (2016) 46: 3338 3343.

[13] Sasson NJ, Dichter GS, Bodfish JW (2012) Affective Responses by Adults with Autism Are Reduced to Social Images but Elevated to Images Related to Circumscribed $\begin{array}{lllll}\text { Interests. PLoS ONE } 7 \text { (8): e42457. doi: } & \end{array}$ 10.1371/journal.pone.0042457.

[14] Gross TF. The perception of four basic emotions in human and nonhuman faces by children with autism and other developmental disabilities. J Abnorm Child Psychol. 2004 Oct; 32 (5): 469-80. 
[15] R. L. Mosley et al. Lost for emotion words: What motor and limbic brain activity reveals about autism and semantic theory. NeuroImage 104 (2015) 413-422.

[16] $\mathrm{Xu}$ et al. Structure of the alexithymic brain: A parametric coordinate-based metaanalysis. Neuroscience and Biobehavioral Reviews 87 (2018) 50-55.
[17] Moriguchi Y, Komaki G. Neuroimaging studies of alexithymia: physical, affective, and social perspectives. BioPsychoSocial Medicine 2013, 7: 8. 